\title{
A Tutorial for an Interactive Narrative Simulation for Alzheimer's Disease's Caregivers: Development and Evaluation
}

\author{
Emilie LETTRY $^{\mathrm{a}, 1}$, Frederic EHRLER ${ }^{\mathrm{b}}$ and Nicolas SZILAS ${ }^{\mathrm{a}}$ \\ ${ }^{\mathrm{a}}$ Tecfa, FPSE, Université de Genève, Switzerland \\ ${ }^{\mathrm{b}}$ University Hospitals of Geneva, Switzerland
}

\begin{abstract}
Alzheimer Care Trainer (ACT) is an interactive narrative simulation for caregivers of patients with Alzheimer's disease. It aims to support family caregivers by letting them practice difficult situations of daily life in a training environment. In order to ensure its accessibility to its target audience, which may be unfamiliar with new technologies, we have created a tutorial. The aim of the tutorial is to teach users the main mechanics of the simulation. Considered to be an introductory level to $\mathrm{ACT}$, it is built in 2 parts, a dirigiste first part and an exploratory, second part. Several tutorial elements, such as context-sensitive information or a help page, have been integrated. The evaluation of the tutorial by 14 participants revealed that interaction mechanics were well understood although the state of the visual element remains confusing. Generated positive affects were stronger than the negative ones and most of the participants were confident to play the game after the tutorial. Ensuring accessibility to all populations is essential especially when targeting seniors. A well-conceived tutorial has the potential to familiarize users with our intervention, reducing the risk of excluding people that could benefit from it.
\end{abstract}

Keywords. Tutorial, Interactive narrative, Serious game

\section{Introduction}

\subsection{The Alzheimer Care Trainer (ACT) narrative simulation}

Caregivers in charge of Alzheimer's patients may feel overwhelmed to provide care on a daily basis. Since the illness is constantly evolving, it is difficult for the caregivers to identify adequate and sustainable behaviour. They become at risk of exhaustion and health issues [7]. In order to deal with this situation we have developed ACT. ACT is a narrative simulation offering a safe training environment allowing caregivers to practice daily care situations to find the most adequate.

The target users of this simulation are informal caregivers such as family (spouses, children), neighbors or friends who are likely to be older than 60 years and may experience age-related cognitive (e.g., decreased cognitive speed) or physical (e.g., visual impairment or hearing loss) disorders [3]. They may be unfamiliar with new technologies, especially computers. They play little or not at all video games.

${ }^{1}$ Corresponding Author, Emilie LETTRY, Tecfa, FPSE, Université de Genève, 24 rue du GénéralDufour CH - 1211 Genève, Switzerland; E-mail: emilie.lettry@gmail.com. 
ACT interface was designed to be as simple as possible in order to ensure accessibility [10]. The player only needs to point and click and does not control the character and its movements, nor manages the camera. The player must click on objects in the $3 \mathrm{D}$ environment or on other characters to interact with them and trigger character actions (see figure 1).

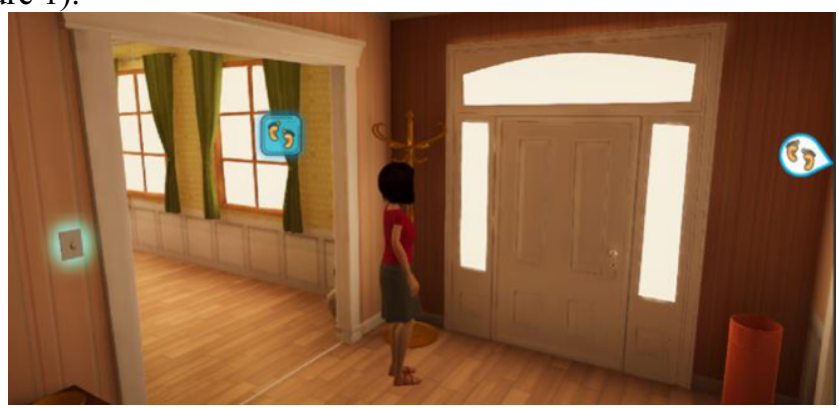

Figure 1. Screenshot of ACT interactive narrative

Despite this simple interface, usability experts highlighted the need for instructions to guide users in getting started with ACT in order to maximize accessibility, this led to the design of a tutorial. Its educational goal is therefore different from ACT itself, as it is limited to the mastery of the interface's commands of the $3 \mathrm{D}$ simulation.

\subsection{Tutorials}

According to Shannon, Boyce, Gadwal and Barnes [9], the main purpose of a tutorial is to familiarize users with the goals and interface of the game. This minimizes the time needed to understand how to use the system so that they can focus and concentrate on the instructional content.

White [11] describes two main ways to teach through a tutorial. First, tutorials that follow a didactic method provide specific instructions that players must follow rigorously. In video games, this takes the form of objectives that appear on the map, instructions from non-player characters, flashcards indicating commands, etc. White [11] refers to this type of tutorial as "worked examples" and states that this type of tutorial is essential for beginners. On the contrary, exploratory tutorials encourage players to play in a non-invasive way and without instructions. In this latter type of tutorials, players must experiment, explore, and make mistakes in order to learn. Shannon, Boyce, Gadwal and Barnes [9] point out that "free play" is not suitable for novices who are unfamiliar with the subject of the game. by the targeted audience

Thus, our objective was to create a tutorial, which familiarizes players with the interface and mechanics of ACT interactive narrative, without eliciting negative reactions, and which adapts to the potential (cognitive, perceptual or health) issues, linked to the target audience age.

\section{Method}

The design of ACT tutorial started with the selection of the educational objectives. Then the flow of the tutorial was defined based on the best practice of the literature in order to ensure a smooth learning curve. The tutorial was then prototyped using screenshots of 
the game with Powerpoint. This process was iterative. As soon as a version of the prototype was ready, it was presented informally to 3 users to collect their feedback and taken into account in the next iteration. This process stopped at the $3 \mathrm{rd}$ iteration, which elicited no more comments regarding the tutorial.

The tutorial was then developed in the game environment, on Unity, and tested online (due to the 2020 pandemic situation)healthy individuals in the same age group as our targeted audience . Participants were recruited and paid by means of a microtasking platform (prolific.co). They had to complete the tutorial online and fill a 20-min questionnaire provided through Qualtrics. The questions aimed at assessing the ease of use and understanding of the tutorial. Moreover, four scales were taken from the IRIS evaluation toolkit [8] in order to assess curiosity, enjoyment, usability and affects.

\section{Results}

\subsection{Learning objectives}

Firstly, the tutorial should familiarize the player with the interactions with the objects in the game. These objects can be in three states of visibility. First, the object can be fully visible. It is therefore indicated by a blueish halo around it. The object can be hidden behind another background object. In this case, the interactive object is highlighted by a blueish square containing a symbol representing the object. Thirdly, the object may be out of the camera field. It is therefore indicated by a white bubble framed in blue and also containing a symbol representing the object. The tip of the bubble indicates the direction of the object (see figure 2).

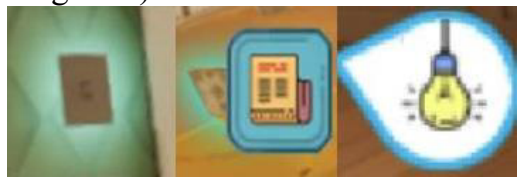

Figure 2. Three states of visibility of objects (fully visible, hidden, out of field)

Secondly, the tutorial must familiarize the player with the interactions with the character representing the patient. When the player clicks on the patient character, a menu containing several interaction options (say, ask, offer help, etc.) representing different ways to interact with the patient appears. The player must then choose one way of interacting from the menu to trigger the interaction. Finally, the tutorial should familiarize the player with moving around the house. To do this, the player must click on the icon representing movements and choose the destination.

\subsection{Tutorial description}

The tutorial is made in two parts, according to a good practice identified by Shannon, Boyce, Gadwal and Barnes [9], i.e. a directed exercise that becomes "free play". The first part is dirigiste [11]. The player must successively perform all the required actions to progress in the tutorial scenario. This aims at ensuring the player has seen and executed two to three times each of the main mechanics of the interactive narrative (interactions with objects in the three states of visibility, dialogue with the patient character, movements). The second part of the tutorial is exploratory, in the form of a sandbox. In this part, all the actions seen in part 1 , as well as new dialogues, become available 
simultaneously, similarly to the real environment of the game. The goal of this part is to provide a safe environment in which the player can learn at her own pace [6] and freely explore the game and its mechanics.

In order to promote the player's immersion in the game as much as possible, instructions are given by the dialogues between the characters, in the dialogue balloons. This follows the customization principle of Clark and Mayer [1], adapted by White [11] for video games and their tutorials. Still in order to maintain this immersion, instructions are not given directly. They are formulated in such a way as to give the player a goal, without telling her how to achieve it. The player has to find out for herself how to achieve it. For example, for the first step of the tutorial, the player is asked to turn on the light by clicking on a switch with the dialogue "It's dark here, you can't see anything" from the patient character.

In case the player is unable to understand the instructions, a contextual information window ("Context-Sensitive information" [2]) is displayed after a few seconds of inactivity of the player. This window explains how to trigger the requested action. The purpose of this page is to provide assistance to players when it seems necessary. This is intended to provide just-in-time information (Gee, 2007, cited by Hedges [4]).

The sandbox section contains a help window accessible by a question mark icon. This 3-pages help window contains a short written paragraph of explanation for each main mechanics, as well as some information about the interface/menu that is not covered in the first part of the tutorial. This aims at formalizing the pedagogical elements discussed in the first part for players who might need it.

Finally, during the dialogic interactions, the player has the choice between 5 dialog options. They represent different ways of addressing the patient (ask, ask to do, say, offer to help, encourage with humor). In order to reduce the cognitive load that these 5 options could represent, the number of options is initially reduced to one during the first dialogue interaction, then gradually expanded to three during the second and third dialogues. The five options become fully available in the second part of the tutorial, the sandbox. This intends to follow the segmentation principle of Clark and Mayer [1], according to which the game mechanics should be taught step by step.

\subsection{User tests}

14 participants belonging to the targeted age range (mean age $=62.5$ years old \pm 2.84 ) were recruited via a crowdsourcing platform. The completion of the selected scale of the IRIS evaluation toolkit showed that the participants felt slightly curious $(\mathrm{m}=3.41$, on a scale from 1- strongly disagree to 5- strongly agree), enjoyment was neutral ( $\mathrm{m}=3.41$, on a scale from 1 - strongly disagree to 5- strongly agree), negative affects were low (m $=2.91$, on a scale from 1 to 10$)$, positive affects were higher $(\mathrm{m}=4.23$, on a scale from 1 to 10$)$ and usability was assessed as neutral $(\mathrm{m}=3.43$, on a scale from 1 - strongly disagree to 5- strongly agree). The highest positive affects items are "interested" $(\mathrm{m}=$ 6.14), "alert" $(\mathrm{m}=6.14)$ and "determined" $(\mathrm{m}=5.29)$. The highest negative affects items are "careful" $(\mathrm{m}=5.71)$ and "sad" $(\mathrm{m}=4.71)$.

In terms of the understanding of the four game mechanics, three of them were fully understood (100\% right answers). The one concerning the visibility states of the game objects was much more problematic (14.29\% right answers). Nevertheless, most of the players $(85.71 \%)$ said they felt confident about playing the narrative game after doing the tutorial. $64.29 \%$ of the participants said they enjoyed the freeplay part of the tutorial. 
$92.86 \%$ of the participants answered "yes" to the question "I always understood what I had to do to keep going in the tutorial".

Table 1. Result of the evaluation

\begin{tabular}{llll}
\hline \multicolumn{1}{c}{ Evaluated item } & & Mean & SD \\
\hline Curiosity (1..5) & 3.41 & 0.99 & \\
Enjoyment (1..5) & 3.41 & 1.00 & \\
Usability (1..5) & 3.43 & 1.1 & \\
Negative affect (1..10) & 2.91 & 1.84 & \\
$\quad$ Careful & 5.71 & 2.23 & \\
Sad & 4.71 & 2.49 \\
Positive affect (1..10) & 4.23 & 2.50 & \\
$\quad$ Interested & 6.14 & 2.54 & \\
Alert & 6.14 & 2.54 & \\
Determined & 5.29 & 2.92 & \\
\hline
\end{tabular}

\section{Discussion and Conclusion}

$\mathrm{ACT}$ is an educational simulation that has the capability to support informal caregivers to deal with daily life situations with Alzheimer patients. Since the target audience is often unfamiliar with new technologies it is important to ensure its accessibility, a key factor for and therefore acceptance [5]. This constraint has moved the design away from well-established design standards, which in return also raised specific usability issues. Therefore a tutorial, with a dedicated learning time on it appears a necessary component. Its design must follow the best practices, which was in our case a two-step approach where the user is challenged appropriately at each stage of the tutorial.

\section{References}

[1] Clark RC, Mayer RE. e-Learning and the Science of Instruction: Proven Guidelines for Consumers and Designers of Multimedia Learning. 2nd ed. San Francisco: Pfeiffer; 2008.

[2] ExtraCredits. Tutorials That Don't Talk Down To You - Context Sensitive Design - Extra Credits. novembre 14, 2018. https://www.youtube.com/watch?v=hFdEEzgc7pg (accessed janvier 2020).

[3] Gerling KM, Schulte FP, Smeddinck J, Masuch M. Game Design for Older Adults: Effects of AgeRelated Changes on Structural Elements of Digital Games. In: Herrlich M, Malaka R, Masuch M, editors. Entertainment Computing - ICEC 2012. Berlin, Heidelberg: Springer; 2012. p. 235-42. (Lecture Notes in Computer Science; vol. 7522).

[4] Hedges, Nathan. Video Game Tutorials: How Do They Teach? Octobre 13, 2017. https://www.gamasutra.com/blogs/NathanHedges/20171013/307378/Video_Game_Tutorials_How_Do They_Teach.php (accessed 20 janvier 2020).

[5] Jia P, Lu Y, Wajda B. Designing for Technology Acceptance in an Ageing Society through Multistakeholder Collaboration. Procedia Manuf [Internet]. 2015;3:3535-42.

[6] Okko, Z. Playful Teaching: How to Create Fun and Immersive Tutorials. october 23, 2017. https://www.gamasutra.com/blogs/ZeinOkko/20171023/308075/Playful_Teaching_How_to_Create_Fu n_and_Immersive_Tutorials.php (accessed January 2020).

[7] Riffin C, Van Ness PH, Wolff JL, Fried T. Family and Other Unpaid Caregivers and Older Adults with and without Dementia and Disability. J Am Geriatr Soc [Internet]. 2017;65(8):1821-8.

[8] Roth C. Experiencing Interactive Storytelling. VU University Amsterdam; 2015.

[9] Shannon A, Boyce A, Gadwal C, Barnes DT. Effective Practices in Game Tutorial Systems. In: Foundations of Digital Games. 2013.

[10] Szilas N, Andkjaer KI, Kemp L, Ricci A, Dadema T, Nap HH, et al. Designing a Senior Friendly Interface for a Personalized 3D Narrative Simulation. In: Foundations of Digital Games. 2020.

[11] White MM. Learn to play: Designing tutorials for video games. Learn to Play: Designing Tutorials for Video Games. New York: A K Peters/CRC Press; 2014. 